\title{
Intestinal glucocorticoid synthesis enzymes in pediatric inflammatory bowel disease patients
}

\author{
Asma Ahmed $^{1,2} \cdot$ Juliane Schwaderer $^{1} \cdot$ Annika Hantusch $^{1} \cdot$ Kaija-Leena Kolho $^{3,4} \cdot$ Thomas Brunner $^{1}$
}

\begin{abstract}
Inflammatory bowel disease (IBD), such as Crohn's disease and ulcerative colitis are devastating chronic immunopathologies of the intestinal mucosa, which are frequently treated by immunosuppressive glucocorticoids. Endogenous glucocorticoids are not only produced by the adrenal glands, but also by the intestinal epithelium. Local glucocorticoid synthesis critically contributes to the immune homeostasis of the intestinal mucosa. As defective intestinal glucocorticoid synthesis has been associated with the development of IBD, we investigated the expression of steroidogenic enzymes and the key transcriptional regulator Liver Receptor Homolog-1 (LRH-1/NR5A2) in ileal and colonic biopsies human pediatric IBD and control patients. Furthermore, the induction of steroidogenic enzymes and their transcriptional regulation by LRH-1 was investigated in a mouse model of experimental colitis. These analyses revealed that colitis-induced expression of steroidogenic enzymes in the murine colon is dependent on the presence of LRH-1, as intestinal deletion of LRH-1 strongly reduced their colitis-induced expression. Similarly, a strong correlation between the expression of LRH-1 and different steroidogenic enzymes was seen in intestinal biopsies of human pediatric patients. Importantly, reduced expression of hydroxysteroid dehydrogenase 11B1 (HSD11B1) was observed in IBD patients compared to control patients, suggesting that defective local reactivation of glucocorticoids could contribute to the pathogenesis of IBD.
\end{abstract}

\section{Introduction}

Inflammatory bowel disease (IBD), i.e. Crohn's disease (CD) and ulcerative colitis (UC), is characterized by a destructive chronic inflammation of the intestinal mucosa $[1,2]$. While much progress has been made in the identification of relevant signaling pathways and gene products involved in the pathogenesis of IBD, the etiology of CD and $\mathrm{UC}$ is often undefined, as different underlying molecular

These authors contributed equally: Asma Ahmed, Juliane Schwaderer

Thomas Brunner

thomas.brunner@uni-konstanz.de

1 Division of Biochemical Pharmacology, Department of Biology, University of Konstanz, Konstanz, Germany

2 Faculty of Medicine, Department of Medicine, University of Khartoum, Khartoum, Sudan

3 Faculty of Medical and Life Sciences, University of Tampere and Tampere University Hospital, Tampere, Finland

4 University of Helsinki, Helsinki, Finland and environmental causes may result in a similar clinical outcome. Yet, given the inflammatory nature of these disease entities a clear contribution of uncontrolled immune responses is evident in both forms of IBD [3]. For this reason, immunosuppressive drugs are often and successfully used for the treatment of IBD patients [4]. Amongst the most frequent immunosuppressive drugs used in the treatment of IBD patients are glucocorticoids (GC) [5].

GC bind to its receptor, the glucocorticoid receptor (GR), and induce a variety of signaling processes, including the inhibition of the transcription factors NFKB (nuclear factor $\kappa \mathrm{B})$ and AP-1 (activator protein-1), and associated inhibition of the production of pro-inflammatory cytokines, ultimately leading to a reduction of inflammation and to an amelioration of the disease [6, 7]. Alternatively, GC are also known to mediate immunosuppression via the induction of apoptosis in immune cells. In particular $\mathrm{T}$ lymphocytes are exquisitely sensitive to GC-induced cell death, which requires the GR-induced upregulation of the pro-apoptotic Bcl-2 homolog Bim [8, 9]. As overreactive T cells are also involved in the pathogenesis of IBD, the apoptosismediated reduction of activated immune cells also contributes to an amelioration of the disease. 
GC are not only an important active ingredient of antiinflammatory drugs, but are also endogenous hormones primarily produced in the adrenal cortex in response to emotional, physical, and immunological stress. Endogenous GC play an essential role in the regulation of immune cells and immune responses. This is evidenced by the fact that blocking endogenous GC synthesis or the removal of the adrenal glands in mice results in lethal septic shock after immune cell activation [10]. Endogenous GC are, however, not only produced by the adrenals. The last two decades of research described various tissues capable of producing GC in response to hormonal regulation and/or immunological stress (reviewed in refs. [11-16]). Thus, we were the first investigating local GC synthesis in the intestinal mucosa, and identified the intestinal epithelium as an important source of immunoregulatory GC [17]. Intestinal GC synthesis was induced upon $\mathrm{T}$ cell and macrophage activation, and required immune cell-expressed TNF (Tumor Necrosis Factor) [18, 19]. This is in as much surprising as TNF is also critically involved in the pathogenesis of IBD and thus a prime target in the therapeutic treatment of IBD patients, as evidenced by the therapeutic effect of TNFneutralizing antibodies [4, 20-22]. In the intestinal mucosa, TNF seem to be also an important sensor of inflammation, and mediate also anti-inflammatory activities via the induction of intestinal GC synthesis [18, 19].

Intestinal GC synthesis and associated transcriptional control of steroidogenic enzymes is regulated by the nuclear receptor family of transcription factors. While adrenal GC synthesis is mainly regulated by the nuclear receptor SF-1 (Steroidogenic Factor-1, NR5A1), its expression is absent in the intestinal epithelium and functionally replaced by its close homolog LRH-1 (Liver Receptor Homolog-1, NR5A2). LRH-1 is expressed along the entero-hepatic axis. In the intestine, it is an important regulator of the intestinal epithelial permeability and immune homeostasis. On one hand, it regulates intestinal epithelial cell stemness and proliferation via the induction of cyclin genes [23], on the other hand it transcriptionally controls the expression of steroidogenic enzymes and the synthesis of GC, and thereby immune homeostasis and inflammation [24]. The role of intestinal GC synthesis in the pathogenesis of experimental IBD was recently investigated. TNF was identified as a critical sensor of inflammation and inducer of intestinal GC. In the absence of TNF a more aggressive form of experimental colitis was observed, and therapeutic administration of TNF restored intestinal GC synthesis and ameliorated colitis [19]. Along these lines we have seen that absence of LRH-1 results in defective intestinal GC synthesis and increased susceptibility to experimental colitis [24, 25]. In support of a role of LRH-1 and intestinal GC synthesis in human patients with IBD, we noticed a negative correlation between steroidogenic factors and pro-inflammatory cytokines in IBD patient samples [25]. Furthermore, intestinal GC synthesis has also been shown to control colonic PPAR $\gamma$ (peroxisome proliferator-activated receptor$\gamma)$ expression. PPAR $\gamma$ plays an important role in the regulation of intestinal immune homeostasis and impaired PPAR $\gamma$ expression has been reported in IBD [26].

Thus, these findings further support a role of intestinal GC synthesis in the regulation of local tissue homeostasis, and suggest that LRH-1 and associated intestinal GC synthesis could be an interesting therapeutic target in patients with IBD. While our experiments with TNF provided a proof-of-principle for such a strategy [19], drugs directly targeting LRH-1 in intestinal epithelium may be a better option [27], as it may stimulate the anti-inflammatory activities of LRH-1 and at the same time the recovery of the epithelium by stimulating proliferation. Though current research suggests that LRH-1 and intestinal GC synthesis in IBD may be indeed an interesting target, still relatively little is known about the intestinal expression of steroidogenic enzymes during IBD, in particular in pediatric patients. The aim of this study therefore was to analyze the expression of LRH-1, steroidogenic enzymes and pro-inflammatory cytokines in pediatric control patients and patients with IBD and compare the findings to non-IBD controls. The data reveal a strong positive correlation between LRH-1 expression and that of the steroidogenic enzymes CYP11A1 and CYP11B1. Furthermore, IBD patients expressed significantly reduced levels of the steroidogenic enzyme HSD11B1 (hydroxysteroid dehydrogenase 11B1), indicating that defective intestinal GC synthesis could indeed contribute to the pathogenesis of IBD. These patients-based data were further confirmed by experimental data in mice, which revealed a clear induction of steroidogenic enzyme expression and intestinal GC synthesis during experimental colitis in an LRH-1-dependent manner. In summary, these data support the notion that LRH-1-regulated intestinal GC synthesis plays an important role in the regulation of the pathogenesis of IBD, in mice and man, and suggest that LRH-1 and associated steroidogenesis in the gut may represent a novel therapeutic target in the treatment of IBD.

\section{Results}

\section{Experimental colitis induces GC synthesis in the murine intestinal mucosa}

We previously described that intestinal immune cell activation and inflammation promotes the local synthesis of GC in mice, which in turn provide a negative feedback, and suppress intestinal immune cells and associated inflammatory processes $[17-19,28]$. In order to investigate these processes in human patients with IBD, we first aimed at 
reanalyzing these processes in a murine model of experimental colitis. Intestinal inflammation was induced by $2.2 \%$ dextran sodium sulfate (DSS) in the drinking water for 5 days followed by 2 days of normal drinking water. DSS leads to a damage of the intestinal epithelium, increased epithelial permeability, and a hyperreaction of the intestinal immune system to the colonic microbiome. Typically, this leads to an acute inflammation, which peaks at day 7 and subsequently resolves [19]. Induction of colonic inflammation and epithelial damage is associated with malabsorption and associated weight loss (Fig. 1a). This is paralleled by a shortening of the colon (Fig. 1b) and an increase of the clinical colitis score (Fig. 1c). We isolated colonic tissue at the onset and the peak of inflammation at day 5 and 7, respectively, and analyzed the expression of steroidogenic enzymes and the key nuclear receptor, LRH1, which regulates their transcriptional expression in the intestinal epithelium [24, 25]. In agreement with previous findings [24], we observed increased expression of LRH-1 at day 5 and 7 post colitis induction (Fig. 1e). In line with the upregulation and activation of LRH-1, we further observed a profound induction of the LRH-1 target genes encoding for the steroidogenic enzymes Cyp11a1 and Cyp11b1, and a moderate upregulation of Cyp21 (Fig. 1f$\mathrm{h}$ ), all involved in the de novo synthesis of corticosterone from cholesterol. Interestingly, we also monitored a colitisdriven upregulation of Hsd11b1, the enzyme involved in the reactivation of corticosterone from inactive dehydrocorticosterone, whereas the corticosterone-inactivating enzyme and counterplayer of Hsd11b1, Hsd11b2, was transiently upregulated at day 5 , but significantly downregulated at day 7 post colitis induction (Fig.1i, j). In general, lower colonic levels of Hsd11b1 were observed, compared to Hsd11b2, which has been observed already previously [29, 30]. These findings confirm that intestinal inflammation drives the expression of steroidogenic enzymes in order to control the local inflammation by the release of immunoregulatory GC. Accordingly, an inflammation-driven increase in colonic GC synthesis was observed (Fig. 1k).

\section{LRH-1-dependent expression of steroidogenic enzymes in the colonic mucosa}

We next investigated the role of LRH-1 in the regulation of basal and colitis-induced expression levels of steroidogenic enzymes. Colitis was thus induced in wild type mice and mice with an intestinal epithelium-specific deletion of LRH1 (villin-cre $\times$ LRH-1 ${ }^{\mathrm{L} / \mathrm{L}}$ ) [25], and the expression of steroidogenic factors was analyzed at day 7 . In line with the previously described role of LRH-1 in the regulation of intestinal inflammation [25, 31], a significantly increased inflammatory response was observed in conditional knockout animals compared to control littermates, as evidenced by increased colon shortening (Fig. 2a), colitis score (Fig. 2b), and colonic histology (Fig. 2c). While we have seen an upregulation of LRH-1 at day 5 after colitis induction (Fig. 1e), no induction was now evident in wildtype animals at day 7 (Fig. 2a), likely due to TNF-mediated downregulation of LRH-1 [31]. In agreement with the deletion of LRH-1 in the intestinal epithelium, even lower levels of LRH-1 expression were seen in conditional knockout. Of interest, LRH-1 expression was not completely absent, suggesting that the remaining LRH-1 expression is derived from a different cellular source, e.g. as previously shown by us for T cells [32] and others for macrophages [33]. While the inflammation-driven induction of the steroidogenic enzymes Cyp11b1, Cyp11a1 and Cyp21 was evident in control animals, epithelial-specific deletion of LRH-1 resulted in a significant reduction of the expression of these enzymes (Fig. 2b-d). In contrast, while a clear colitis-induced upregulation of Hsd11b1 and a downregulation of Hsd11b2 was observed, no differences between control animals and conditional knockouts were evident (Fig. 2e, f).

\section{Expression of steroidogenic factors in pediatric human ileal and colonic tissue biopsies}

The colitis experiments in mice described above clearly confirmed that (i) colitis induces the upregulation of steroidogenic enzymes and the colonic synthesis of GC, and that (ii) the expression of the steroidogenic cytochrome P450 (Cyp) enzymes is critically controlled by epithelial LRH-1 expression. In order to establish a correlation between LRH-1 expression and intestinal steroidogenesis also in the human intestinal mucosa, we monitored the expression of relevant steroidogenic factors in human pediatric intestinal biopsies. Ileal and colonic tissue samples from IBD and non-IBD controls (no known intestinal pathology) were analyzed for the expression of LRH-1 (NR5A2), the steroidogenic enzymes CYP11A1, CYP21A1, HSD11B1, and HSD11B2, and IBD-associated pro-inflammatory cytokine TNF. The number of samples included in the analysis varied, as when the CT values in the quantitative PCR were high ( $>32$ ), a considerable variation in the duplicate samples was noticed (standard deviation $>1$ ), and thus these samples were excluded. When all tissue samples were combined in the statistical analysis of gene expression a clear positive correlation was observed for ileal expression of LRH-1 and CYP11A1, and LRH-1 and CYP21A1 (Fig. 3a). This positive correlation between the expression of LRH-1 and these steroidogenic enzymes was also confirmed in colonic tissue samples (Fig. 3b). In addition, a positive correlation between colonic LRH-1 expression and HSD11B1 expression was seen (Fig. 3b). 
A

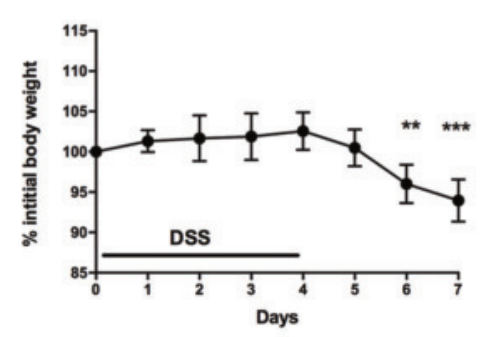

D

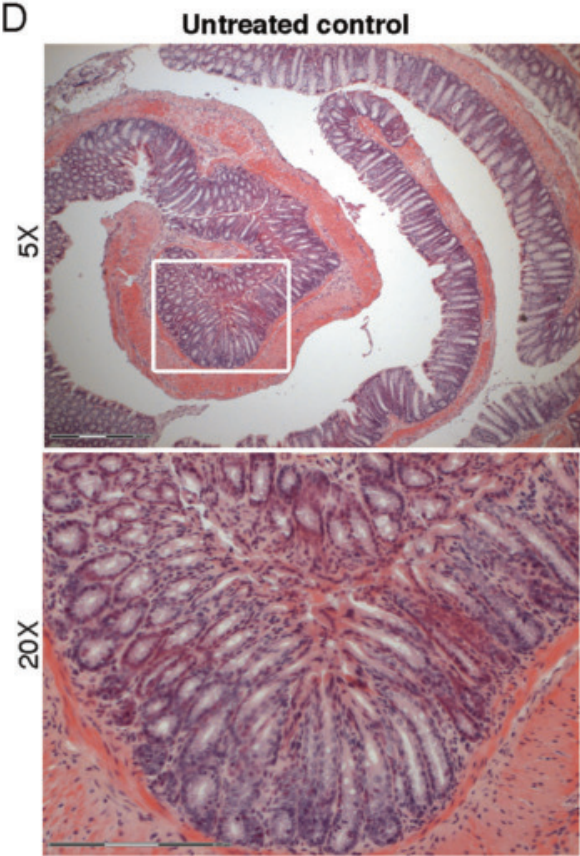

E

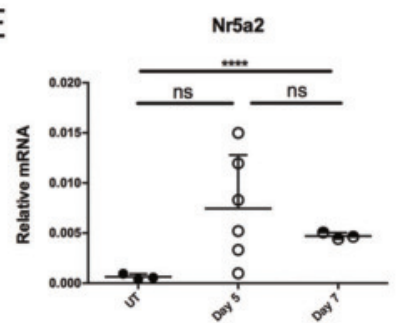

I

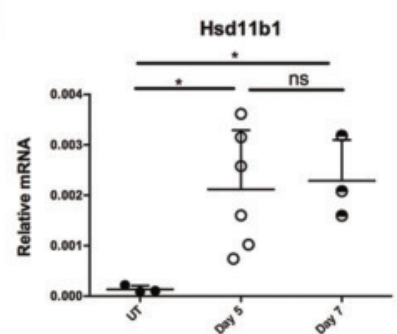

Colon length

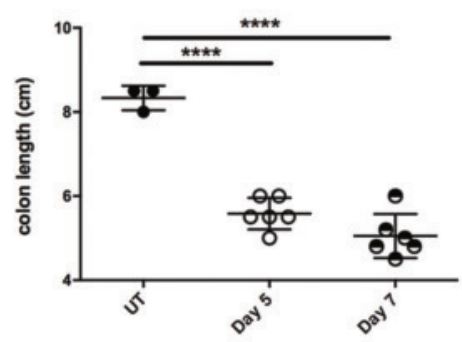

DSS day 5

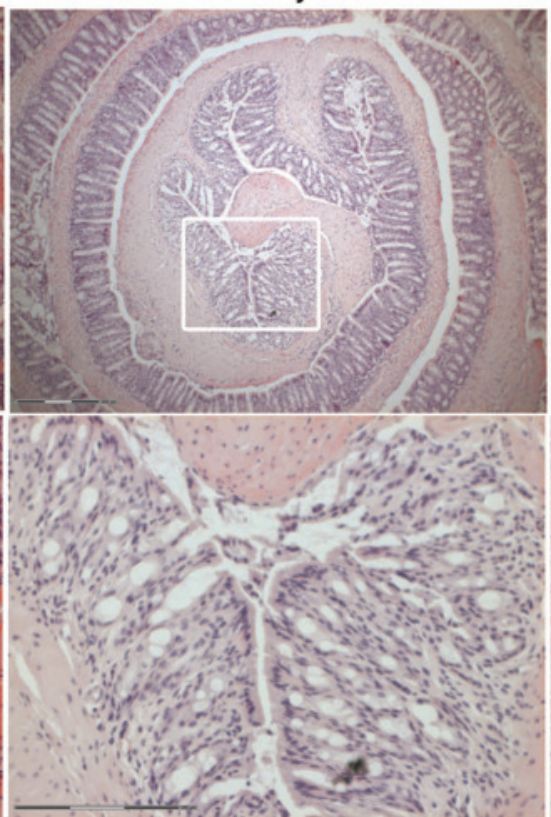

C

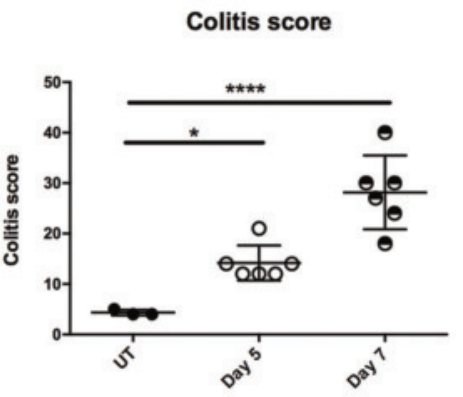

DSS day 7

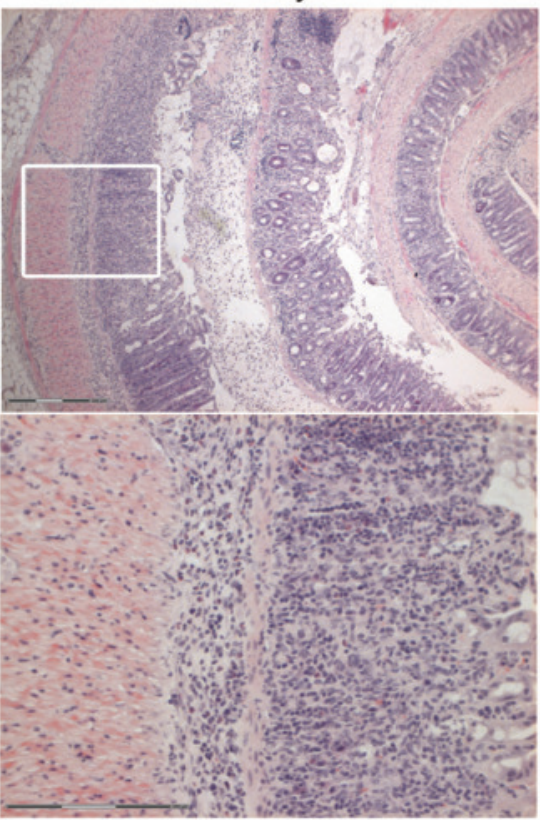

$\mathrm{F}$

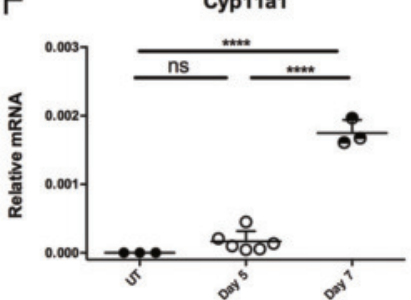

J

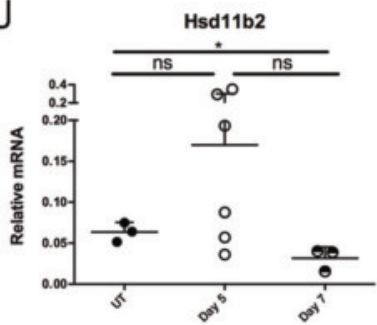

G
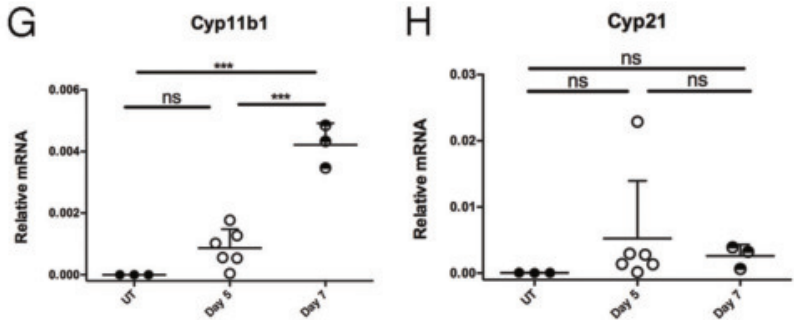

$\mathrm{K}$

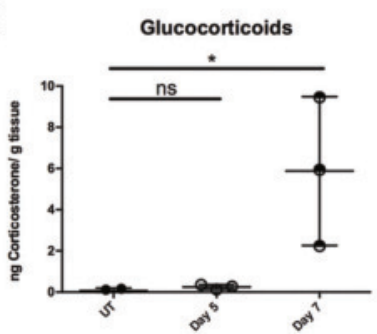

Interestingly, in both tissues a positive correlation between CYP11A1 and CYP21A1, and CYP11A1 and HSD11B1 was seen, indicating that their relative expression is similarly regulated.
The animal experiments described above already revealed a differential regulation of Hsd11b1 and Hsd11b2, the GC-activating enzyme Hsd11b1 being upregulated during colitis, and the GC-inactivating enzyme Hsd11b2 
Fig. 1 Intestinal glucocorticoid synthesis during experimental colitis in mice. Colitis was induced in 7-11 weeks old wild-type mice $(n=12)$ by treatment with $2.2 \%(\mathrm{w} / \mathrm{v})$ DSS in the drinking water for 5 days followed by 2 days of normal drinking water, control mice were treated with normal drinking water $(n=3)$. a Percent body weight change normalized to initial body weight at day zero. b Colon length and $\mathbf{c}$ histological colitis score from untreated control and DSS-treated mice at day 5 and 7. d Representative histological sections of colons from untreated and DSS-treated mice at days 5 and 7 (5X magnification: upper panel, $\times 20$ magnification: lower panel). $\mathbf{e}-\mathbf{j}$ RNA was extracted from colonic tissue of untreated (UT) or DSS-treated mice at day 5 and 7, and analyzed by quantitative PCR. Gene expression of LRH-1 (e), Cyp11a1 (f), Cyp11b1 (g), Cyp21 (h), Hsd11b1 (i) and Hsd11b2 (j) was measured. $\beta$-actin was used as a reference mRNA. Relative mRNA levels are shown $\left(2^{-\Delta \mathrm{Ct}}\right)$. $\mathbf{k}$ Glucocorticoid synthesis in colonic tissue from control (UT) and DSS-treated mice at day 5 and 7 ( $n=3$ per group). $* p<0.05, * * p<0.01, * * * p<0.001, * * * * p<$ 0.0001 . UT untreated, ns not significant

being downregulated. Interestingly, also a negative correlation between ileal, resp. colonic, expression of HSD11B1 and HSD11B2 was seen in human pediatric samples. Similarly, a negative correlation was seen between colonic HSD11B2 and CYP21A1 (Fig. 3a, b).

Somewhat surprisingly, no (positive or negative) correlation was seen between the expression of LRH-1 or steroidogenic enzymes, and the pro-inflammatory cytokine TNF. This may be related to the complex interaction between this cytokine and intestinal steroidogenesis. While TNF promotes inflammation, intestinal GC synthesis suppresses it and thereby also the expression of TNF. On the other hand, TNF is an important inducer of intestinal steroidogenic enzymes and GC synthesis [18, 19]. Thus, TNF and steroidogenic enzymes may be implicated in a complex and circular network of regulation.

\section{Reduced expression of colonic HSD11B1 in IBD patients}

We have previously suggested that altered intestinal GC synthesis may contribute to the pathogenesis of IBD [14, $19,25]$. We thus next compared the relative expression levels of steroidogenic factors in control patients $(n=5-7)$ versus IBD patients. Due to the limited number of patients with defined (Crohn's disease, ulcerative colitis) or undefined pathologies (IBDU), all patients with IBD were pooled in one group, but annotated separately $(n=10-12$ total). In agreement with an inflammation-induced upregulation of steroidogenic factors seen in the animal experiments (Figs. 1 and 2), we noticed a tendency towards increased expression of LRH-1 (NR5A2), CYP11A1 and CYP21A1 in ileal and colonic biopsies from IBD patients, compared to control patients, although this difference was not statistically significant (Fig. 4a, b). In agreement with a potentially increased intestinal GC synthesis during the pathogenesis of IBD, we also observed a reduced ileal expression of TNF in patients with IBD (Fig. 4a). Interestingly, while all CYP enzymes did not show significant differences between control and IBD patients, we found that the GC-activating enzyme HSD11B1 was significantly less expressed in colonic biopsies of IBD patients, compared to control patients, while the counteracting enzyme HSD11B2 showed a tendency towards increased expression (Fig. 4b). Thus, these findings suggest that development of IBD could be associated with defective reactivation of antiinflammatory cortisol from inactive cortisone via an HSD11B1-mediated enzymatic step.

\section{Discussion}

GC are stress hormones with potent anti-inflammatory activities. Therefore, synthetic GC are frequently and successfully used in the treatment of a wide variety of inflammatory diseases, including IBD. While endogenous GC are predominantly produced by the adrenal glands, there is increasing evidence that de novo GC synthesis from cholesterol and/or HSD11B1-mediated reactivation of inactive GC (dehydrocorticosterone, resp. cortisone) also occurs in different extra-adrenal tissues. Examples for such GC-synthesizing tissues are the thymus, skin, lung and, as discussed here, the intestinal mucosa (reviewed in [11, 14, 15]). Various reports indicate that the local synthesis of GC in the intestinal epithelium may be involved in the pathogenesis of intestinal inflammation, i.e. CD and UC [19, 25, $34,35]$. While the regulation and the impact of local GC synthesis on intestinal inflammation has been investigated in some detail in different mouse models of colitis [19, 25, 28], far less is known about these processes in human IBD patients. The goal of this study was therefore to investigate the gene expression pattern of steroidogenic factors in tissue biopsies from healthy controls and IBD patients in order to correlate the expression of the key regulatory transcription factor LRH-1 with that of steroidogenic enzymes. Furthermore, we aimed at investigating whether changes in gene expression could indicate defective intestinal GC synthesis in the pathogenesis of IBD.

Confirming our previous findings in murine [17, 19, 24, $28,36]$ and human intestinal tissue [25, 37], we observed that LRH-1 and various steroidogenic enzymes are expressed at detectable levels in ileal as well as colonic tissue biopsies. Furthermore, the statistical analysis of the expression data demonstrated an obvious correlation between LRH-1 expression and the expression of the steroidogenic enzymes CYP11A1, CYP21A1, and in the colon also HSD11B1 (Fig. 3). This suggests that also in human intestinal tissue LRH-1 has an important role in the transcriptional control of these steroidogenic enzymes, and confirms our previous findings in mice that the intestinal 

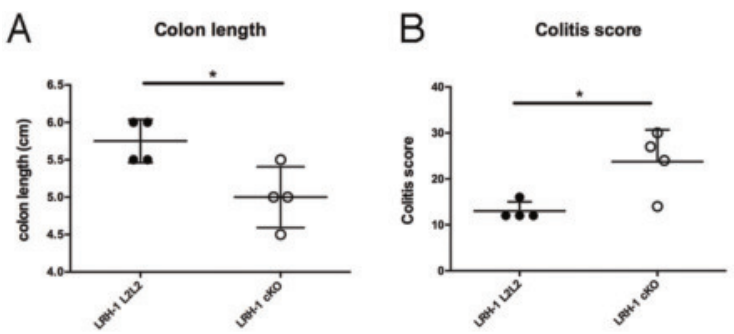

C

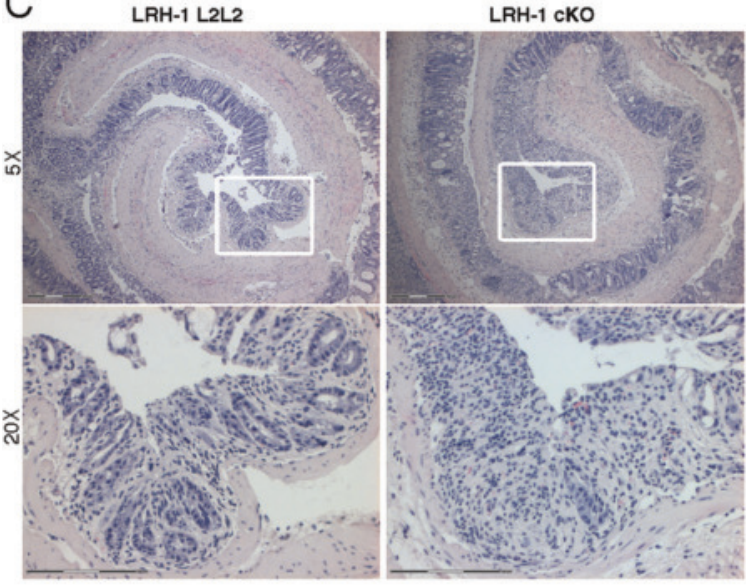

Fig. 2 Role of LRH-1 in the regulation of intestinal steroidogenesis and inflammation. Colitis was induced in wild-type mice (LRH-1 L2/ L2) and mice with intestinal epithelial-specific LRH-1 deletion (LRH$1 \mathrm{cKO})(n=4$ per group) by treatment with $2.2 \%(\mathrm{w} / \mathrm{v}) \mathrm{DSS}$ in the drinking water for 5 days followed by 2 days of normal drinking water. Control mice received normal drinking water $(n=6)$. a Colon length and b histological colitis score at day 7 after DSS treatment. c Representative histological sections of colonic tissues from LRH-1 L2/

GC synthesis is critically controlled by this nuclear receptor [18, 25, 37]. Whereas CYP11B1 and CYP11A1 have been identified as direct transcriptional targets of LRH-1 [38], the role of LRH-1 in the regulation of HSD11B1 is currently less clear. While murine Hsd11b1 is upregulated like Cyp11a1, Cyp11b1, and Cyp21 during experimental colitis in mice (Fig. 1), its induction seems not to be affected by the epithelial-specific deletion of LRH-1, unlike the expression of Cyp11a1, Cyp11b1, and Cyp21. This suggests that Hsd11b1 is regulated by inflammatory signals, possibly also TNF $[18,19]$, but not directly transcriptionally controlled by LRH-1.

While we have been able to show a nice positive correlation between LRH-1 and the expression of multiple steroidogenic enzymes (Fig. 3), interestingly, a negative correlation between the two counterplayers in the inactivation, resp. reactivation of cortisol and cortisone, i.e. HSD11B2 and HSD11B1 was noticed. Last but not least, we have seen a statistically significant difference in the colonic HSD11B1 expression in control biopsies versus tissue samples from IBD patients (Fig. 4b). While these data do not formally prove defective colonic GC synthesis in
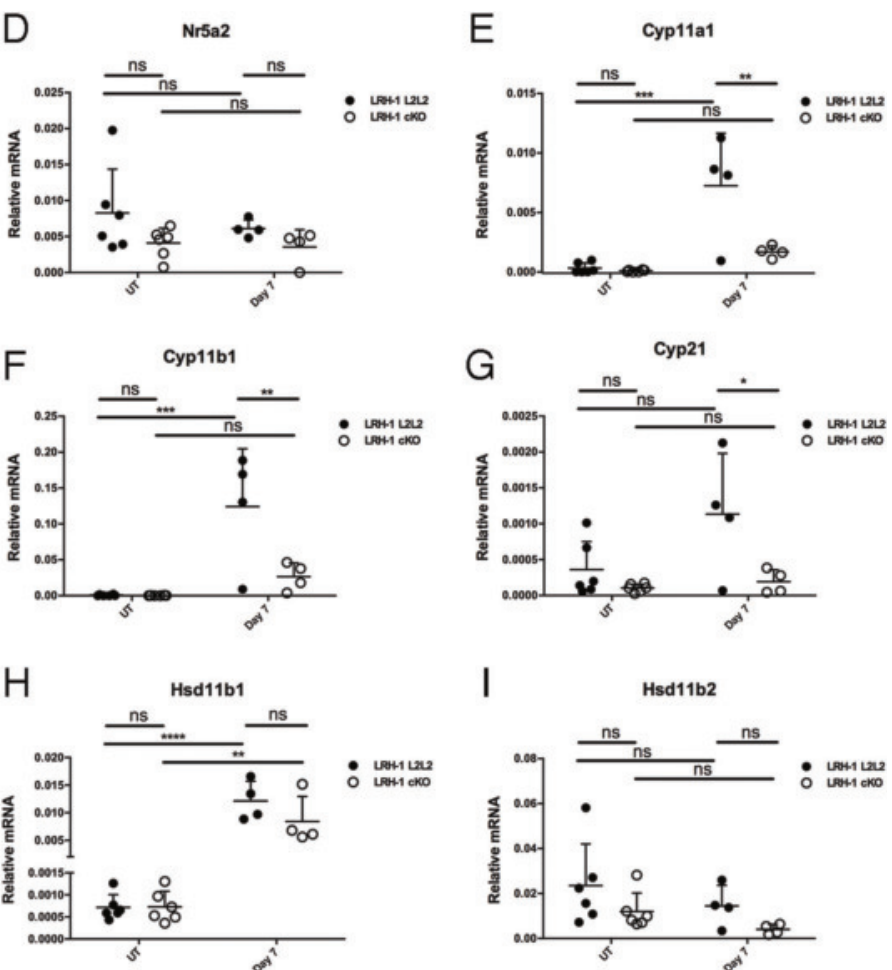

L2 and LRH-1 cKO at day 7 after DSS treatment $(\times 5$ magnification: upper panel, $\times 20$ magnification: lower panel). $\mathbf{d}-\mathbf{i}$ RNA was extracted from colonic tissues of untreated or DSS-treated mice at day 7 and analyzed by quantitative PCR. Gene expression of LRH-1 (d), Cyp11a1 (e), Cyp11b1 (f), Cyp21 (g), Hsd11b1 (h) and Hsd11b2 (i) was measured. Relative mRNA levels are shown $\left(2^{-\Delta \mathrm{Ct}}\right) . * p<0.05$, $* * p<0.01, * * * p<0.001, * * * * p<0.0001$. UT untreated, ns not significant

IBD patients, they suggest that the reduced expression levels of HSD11B1 could result in reduced local reactivation of cortisone to cortisol, and thus limiting local levels of active immunosuppressive GC.

The opposite transcriptional regulation of Hsd11b1 and Hsd11b2 is of interest. While induction of experimental colitis resulted in an upregulation of Hsd11b1, a parallel downregulation of Hsd11b2 was noticed. Interestingly, this expression pattern had previously also been observed in IBD patients [35]. This suggest that next to the induction of de novo GC synthesis, the colonic tissue appears to aim at maintaining high levels of local GC by limiting an Hsd11b2-mediated inactivation while increasing an Hsd11b1-mediated reactivation of GC. While we have previously shown that the release of GC from intestinal tissue biopsies is blockable by the CYP11B 1 and HSD11B1 inhibitor metyrapone [18, 19, 24], allowing to exclude serum contaminations from the measurements, recently Reis de Souza and colleagues [39] investigated the role of adrenal-derived GC in the pathogenesis of experimental colitis. They found that adrenalectomy resulted in reduced release of colonic GC and negatively impacted the 
A

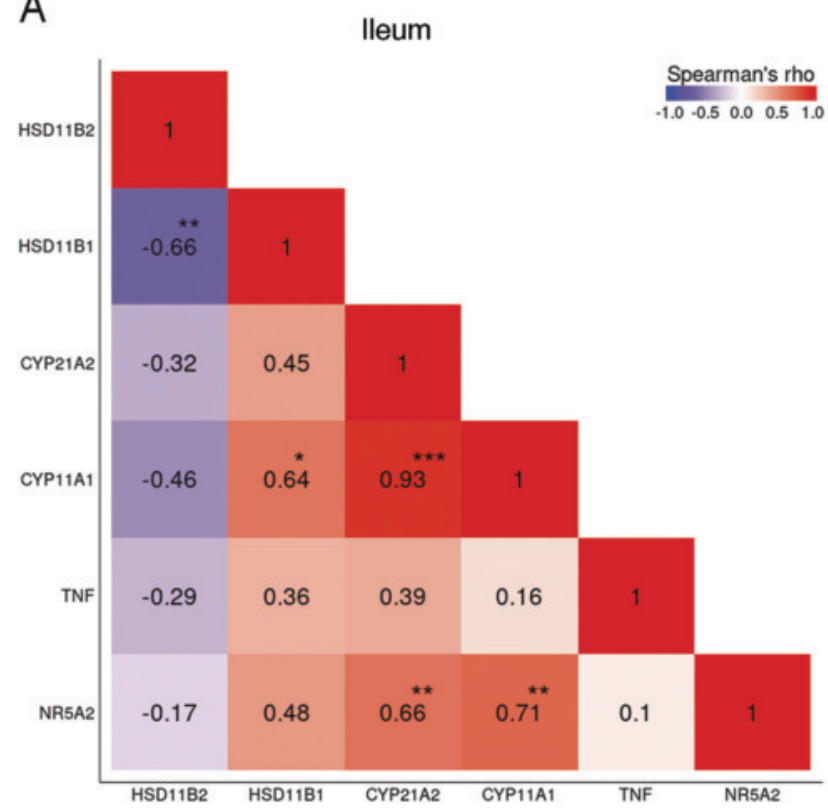

Fig. 3 Correlation between the expression of different steroidogenic enzymes, LRH-1 and TNF, in human intestinal tissue samples. Gene expression in ileal (a) and colonic (b) tissue biopsies from all donors (control group and IBD patients) were analyzed by quantitative PCR. The correlation coefficient for the comparison of gene expression data

pathogenesis of experimental colitis, suggesting that a Hsd11b1-mediated reactivation of dehydrocorticosterone to active corticosterone may contribute to the immune regulation during intestinal inflammation.

Surprisingly, the detailed analysis of our patient cohort compared to the mouse data revealed the opposite finding. Reduced HSD11B1 expression, rather than an increase as during experimental colitis (Fig. 1i), was seen in colonic biopsies of IBD patients (Fig. 4b). A reduced expression of the steroidogenic enzymes CYP11A1 and B1 had also been observed in intestinal samples from adult CD and UC patients, although HSD11B1 had not been reported [25]. This positively correlated with reduced LRH-1 expression and negatively with increased expression of proinflammatory cytokines. This observation may be related to two underlying processes. On one hand, LRH-1 expression and thus that of steroidogenic enzymes appears to be restricted to the intestinal crypts [17, 23, 24]. As severe acute flares of IBD are often associated with a destruction of colonic crypts, the reduced expression of LRH-1 and steroidogenic enzymes may be related to the relative loss of their cellular source. On the other hand, while we have previously shown that during acute immune cell activation and experimental colitis TNF is a key trigger of LRH-1dependent intestinal steroidogenesis [18, 19], it was also noticed that TNF results in reduced LRH-1 expression [31], and reduced intestinal GC synthesis during chronic colitis
B

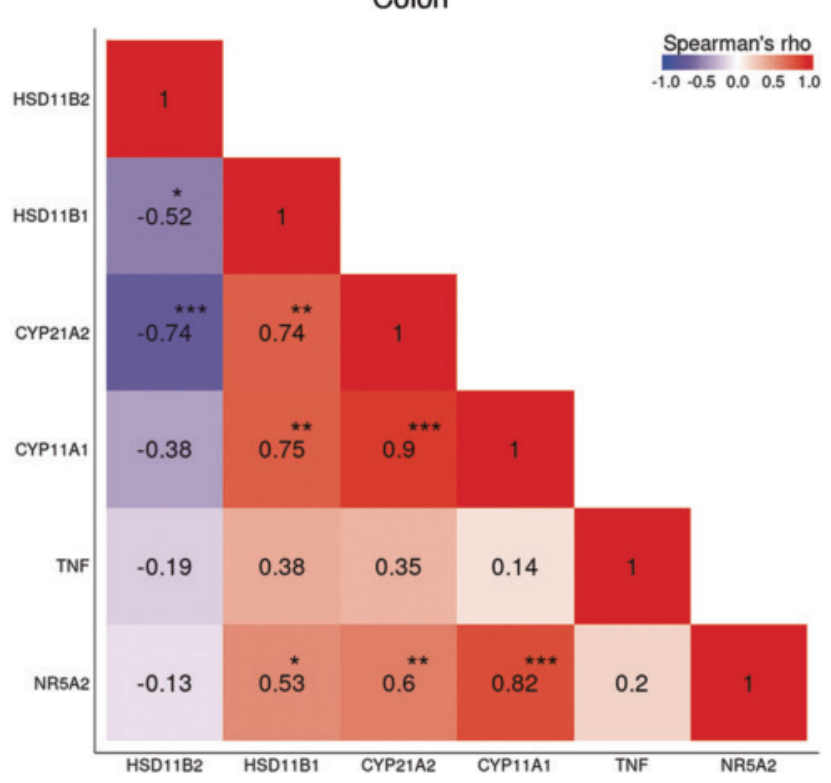

(of $\Delta \mathrm{Ct}$ values, the difference in the PCR cycle numbers between the gene of interest and the house keeping gene) was calculated using Spearman's rank correlation test. The heat map shows color-coded rho values. $* p<0.05, * * p<0.01, * * * p<0.001$

[40]. Thus, depending on the disease state of the patient, opposite expression pattern between TNF on one hand, and LRH-1 and steroidogenic enzymes on the other hand, may be expected. Similarly, the discrepancy between patient data and mouse model may be based on species differences, and/ or chronic versus acute inflammation.

Our data presented here indicate that (i) LRH-1 has a similarly important role in the regulation of intestinal steroidogenesis in mice and men, and that (ii) defective intestinal GC synthesis could contribute to the pathogenesis of IBD in human patients. This illustrates the potential of LRH-1-based therapies in the treatment of IBD patients. Thus far, LRH-1 is still considered an orphan nuclear receptor as no endogenous ligands have been identified yet. However, the ligand-binding pocket of LRH-1 appears to be able to accommodate a variety of lipophilic substances, and many of them enhance the transcriptional activity of LRH1, as would be expected from an LRH-1 ligand [41]. A most interesting molecule in this regard is the phospholipid dilauroyl phosphocholine (DLPC). DLPC had been identified in a chemical screen and shown to enhance LRH-1's transcriptional activity. Importantly, therapeutic administration of DLPC resulted in a significant improvement of a high fat diet-induced LRH-1-regulated lipid accumulation in the liver and associated type 2 diabetes [27]. While the therapeutic effect of DLPC or similar substances have not yet been tested in an experimental model of colitis, it is 
A

Ileum
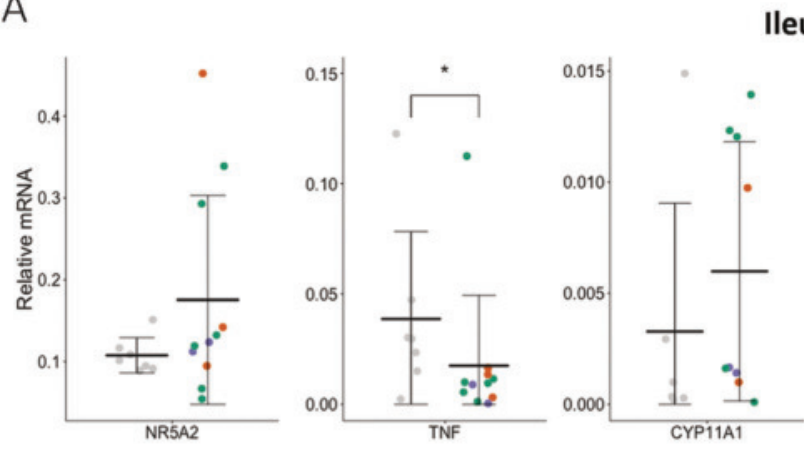

B
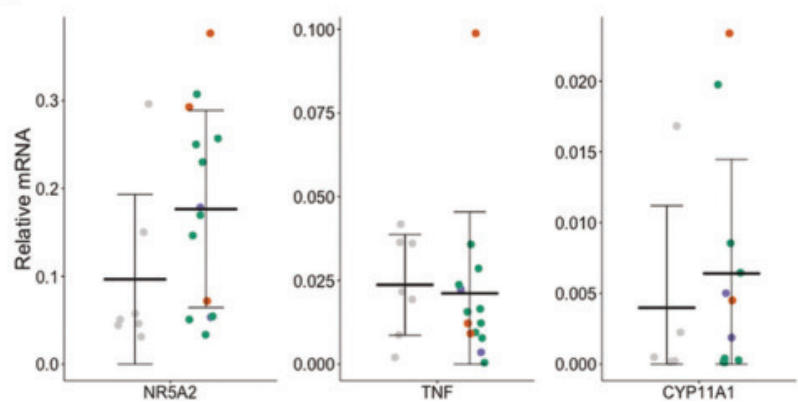

Fig. 4 Comparison of steroidogenic factor expression in control group vs. IBD patients. Gene expression data in ileal (a) and colonic (b) tissue biopsies from control and IBD patients were analyzed by quantitative PCR. Relative mRNA levels are shown $\left(2^{-\Delta \mathrm{Ct}}\right)$. Differences in the expression of individual gene products between control patients and IBD patients were analyzed by Mann-Whitney test.
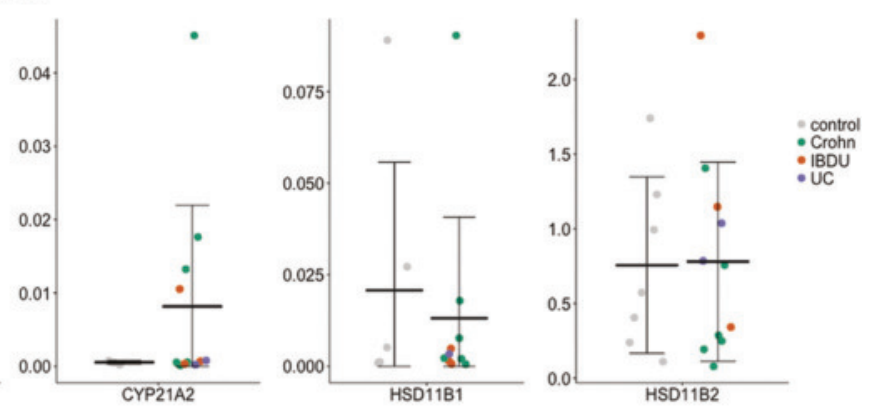

Colon
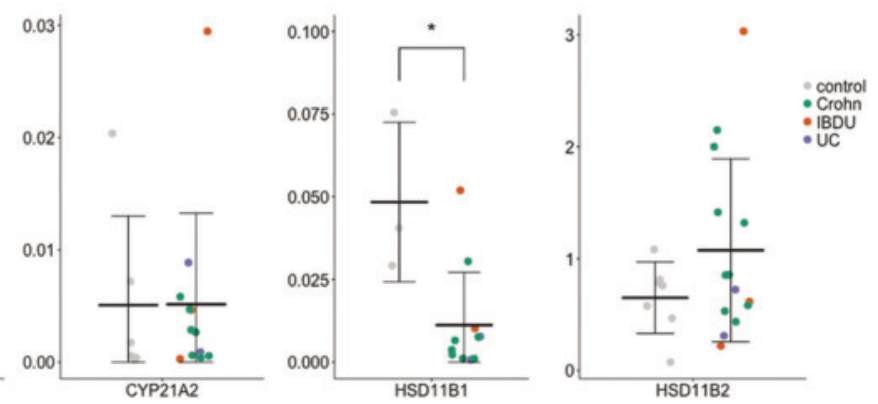

Individual values of patients from the different disease entities (control, Crohn's diease (CD), ulcerative colitis (UC), and undefined IBD (IBDU)) are depicted. Color codes correspond to different disease entities (gray $=$ controls, green $=\mathrm{CD}$, blue $\mathrm{UC}$, red IBDU). Mean values (horizontal lines) and standard deviations (error bars) are shown. ${ }^{*} p<0.05, * * p<0.01, * * * p<0.001$

spectrophotometer (GE Healthcare, Waskesha, WI), and ranged from $50.6-325 \mathrm{ng} / \mu \mathrm{l}$, with a mean of $121 \mathrm{ng} / \mu \mathrm{l}$.

The Ethics committee of Helsinki University Hospital approved the study. All patients and/or their guardians gave an informed consent for the study.

\section{Mice}

\section{Patients and intestinal tissue samples}

Intestinal biopsies from the ileum $(n=19)$ and the colon $(n$ $=22$ ) were obtained during ileocolonscopy performed to diagnose pediatric IBD or for its follow-up or for exclusion of IBD. There were 17 patients with IBD (Crohn's disease $n=10$, UC $n=3$, IBD unclassified, IBDU, $n=4)$ and 8 non-IBD control patients (considered as healthy with no known intestinal pathology). The mean age of the patients was 11.8 years (range $2.4-18$; male 13). The samples were obtained between November 2006 and May 2008 at Children's Hospital, University of Helsinki, a tertiary care center in Finland. Biopsy samples were inserted in vials with RNAlater solution (Qiagen, Hilden, Germany), prior to isolation of RNA. The concentrations of resulting RNA in samples was quantified using a NanoVue
Wild-type C57B1/6 mice, mice with floxed LRH-1 gene $\left(\mathrm{LRH}-1^{\mathrm{L} / \mathrm{L}}\right)$ and mice with conditional deletion of LRH-1 in the intestinal epithelium (villin-Cre $\mathrm{x} \mathrm{LRH}-1^{\mathrm{L} / \mathrm{L}}, \mathrm{cKO}$ ) [25] were bred and housed in the Central Animal Facility of the University of Konstanz in individually ventilated cages under specified pathogen-free conditions. All mice were used at the age of 7-11 weeks, and were age and sex matched.

\section{Induction and analysis of experimental colitis}

DSS (dextran sodium sulfate) colitis was induced in wild type $(n=12), \mathrm{LRH}-1^{\mathrm{L} / \mathrm{L}}$ and cKO mice $(n=4$ per genotype) by administration of $2.2 \%$ (wt/vol) DSS (reagentgrade DSS salt; molecular mass $=36-50 \mathrm{kD}$; MP 
Biomedicals) in normal drinking water for 5 days, followed by 2 days normal water consumption. Control wild-type mice $(n=3)$ and control LRH- $1^{\mathrm{L} / \mathrm{L}}$ and $\mathrm{cKO}(n=6$ per genotype) received normal drinking water. Wild type mice were euthanized at day 5 and 7 ( $n=6$ per time point) and day 7 for LRH-1 $1^{\mathrm{L} / \mathrm{L}}$ and cKO mice ( $n=4$ per genotype). The entire colon was then isolated, length was measured, and then cut longitudinally. One third of the tissue was used for RNA isolation and analysis of gene expression, whereas the remaining two thirds were used for histological analysis and ex vivo culture for measurement of colonic GC synthesis. Histological colitis scoring was performed as described previously [42].

\section{Detection of colonic glucocorticoid synthesis}

Analysis of colonic glucocorticoid synthesis was done as described previously [17-19].

\section{Detection of steroidogenic factors by quantitative PCR}

RNA from murine tissues was isolated using RNA isolation kits (Promega, Mannheim, Germany), and RNA concentration and quality was analyzed on a Nanodrop Spectrophotometer (Thermo Fisher Scietific, Germany). RNA from human and murine tissues was reverse transcribed, and resulting cDNA was analyzed for gene expression on a One-Step-Plus realtime PCR machine (Applied Biosystems, Foster City, CA) using the following primers:

Human primers:

human GAPDH for 5'- ATG GAG AAG GCT GGG GCT CA -3', human GAPDH rev 5'- AGT GAT GGC ATG GAC TGT GGT CAT - $3^{\prime}$, human LRH-1 for 5' - GGG CAA CAA GTG GAC TAT TC -3', human LRH-1 rev 5'- CCA GCT GGA AGT TTT CAA GG -3', human HSD11B2 for 5' - ACA TTA GCC GCG TGC TAG AGT TCA -3', human HSD11B2 rev 5'- CGC GCC AAA GAA ATT CAC CTC CAT -3', human CYP21 for 5'- CCA TCT ATC TGC TTG GCC TGA -3', human CYP21 rev 5'- TAG ACA CCA GCT TGT CTT GCA G -3', human TNF for 5'- TCT TCT CCT TCC TGA TCG TG -3', human TNF rev 5'- GCC AGA GGG CTG ATT AGA GA -3'. Human HSD11B1 and human CYP11A1 primers were obtained from Qiagen (Quantitect primers).

Murine primers:

murine LRH-1 (Nr5a2) for TCA TGC TGC CCA AAG TGG AGA, murine LRH-1 (Nr5a2) rev TGG TTT TGG ACA GTT CGC TT; murine Cyp11a1 for CTT TGA GTC CAT CAG CAG TGT T, murine Cyp11a1 rev TGG TAG ACA GCA TTG ATG AAC C; murine Hsd11b1 for GCC AGC AAA GGG ATT GGA AG, murine Hsd11b1 rev
CGA GTT CAA GGC AGC GAG AC; murine $\beta$-actin for TAT TGG CAA CGA GCG GTT CC, murine $\beta$-actin rev GCA CTG TGT TGG CAT AGA GG.

Murine Cyp11b1, Hsd11b2, and Cyp21 primers were obtained from Qiagen (Quantitect primers).

\section{Statistical analysis of data}

Statistical analysis of mouse data was performed using Prism software version 6 (Graphpad Software, La Jolla CA). Student's $t$-test was used to compare two groups, and ordinary one-way ANOVA was used to compare more than two groups. To compare the quantitative PCR expression data from untreated wild-type control mice vs. DSS-treated mice at days 5 and 7, ordinary one-way ANOVA was used. To compare the quantitative PCR expression data from untreated vs. DSS-treated (LRH-1 ${ }^{\mathrm{L} / \mathrm{L}}$ ) and LRH-1 (villin-cre $\left.\mathrm{x} \mathrm{LRH}^{1}{ }^{1 / \mathrm{L}}\right)$ mice, two-way ANOVA was performed. $* p<$ $0.05, * * p<0.01, * * * p<0.001, * * * * p<0.0001$.

Quantitative PCR expression analysis data of total human samples were analyzed and compared by regression analysis. Spearman's rho values were calculated for the correlation of not normally distributed ileal and colonic expression data from from $\Delta \mathrm{Ct}$ values of control and IBD patients and illustrated in a color-coded heat map. $* p<0.05$, $* * p<0.01, * * * p<0.001$.

To compare expression data from healthy controls versus IBD patients the Mann-Whitney test was used. In the IBD group samples from Crohn's disease and ulcerative colitis patients, and IBD patients with undefined disease entity (IBDU) were included. Samples from control patients that had celiac disease or primary endoscopic biopsies were excluded from statistical analyses. Similarly, samples resulting in an incorrect amplification, aberrant melting curves in the PCR reaction, or $\mathrm{Ct}$ values $>32$ and a standard deviation of duplicate measurements of $>1$ were excluded from the analysis. $* p<0.05, * * p<0.01, * * * p<0.001$.

Acknowledgements We would like to thank Susanna Lintula and Jacob Stenman for performing the RNA extraction of the human biopsy samples, and the members of the Brunner lab for technical support and advice. This work was supported in part by research grants from German Science Foundation to TB, and Pediatric Research Foundation, Finland, and Helsinki University Hospital Research Fund, Finland to KLK. AA was supported by a fellowship from the Ministry of Higher Education and Scientific Research, Sudan and the BadenWürttemberg-Stiftung.

\section{Compliance with ethical standards}

Conflict of interest The authors declare that they have no conflict of interest. 


\section{References}

1. Hanauer SB, Present DH. The state of the art in the management of inflammatory bowel disease. Rev Gastroenterol Disord. 2003;3:81-92.

2. Shaw KA, Cutler DJ, Okou D, Dodd A, Aronow BJ, Haberman Y et al. Genetic variants and pathways implicated in a pediatric inflammatory bowel disease cohort. Genes Immun. 2018. https:// doi.org/10.1038/s41435-018-0015-2. [Epub ahead of print].

3. Powrie F. T cells in inflammatory bowel disease: protective and pathogenic roles. Immunity. 1995;3:171-4.

4. Atreya R, Neurath MF. New therapeutic strategies for treatment of inflammatory bowel disease. Mucosal Immunol. 2008;1:175-82.

5. Cross RK. Safety considerations with the use of corticosteroids and biologic therapies in mild-to-moderate ulcerative colitis. Inflamm Bowel Dis. 2017;23:1689-701.

6. Sapolsky RM, Romero LM, Munck AU. How do glucocorticoids influence stress responses? Integrating permissive, suppressive, stimulatory, and preparative actions. Endocr Rev. 2000;21:55-89.

7. Schaaf MJ, Cidlowski JA. Molecular mechanisms of glucocorticoid action and resistance. J Steroid Biochem Mol Biol. 2002;83:37-48

8. Erlacher M, Michalak EM, Kelly PN, Labi V, Niederegger H, Coultas L, et al. BH3-only proteins Puma and Bim are ratelimiting for gamma-radiation- and glucocorticoid-induced apoptosis of lymphoid cells in vivo. Blood. 2005;106:4131-8.

9. Wang Z, Malone $\mathrm{MH}$, He $\mathrm{H}$, McColl KS, Distelhorst CW. Microarray analysis uncovers the induction of the proapoptotic BH3-only protein Bim in multiple models of glucocorticoidinduced apoptosis. J Biol Chem. 2003;278:23861-7.

10. Gonzalo JA, Gonzalez-Garcia A, Martinez-A C, Kroemer G. Glucocorticoid-mediated control of the activation and clonal deletion of peripheral T cells in vivo. J Exp Med. 1993;177:123946.

11. Taves MD, Gomez-Sanchez CE, Soma KK. Extra-adrenal glucocorticoids and mineralocorticoids: evidence for local synthesis, regulation, and function. Am J Physiol Endocrinol Metab. 2011;301:E11-24.

12. Kostadinova F, Hostettler N, Bianchi P, Brunner T. Extra-adrenal glucocorticoid synthesis in mucosal tissues and its implication in mucosal immune homeostasis and tumour development. In: Qian X (ed.) Glucocorticoids: New Recognition of Our Familiar Friend. Intech: Rijeka, Croatia; 2012.

13. Kostadinova F, Schwaderer J, Sebeo V, Brunner T. Why does the gut synthesize glucocorticoids?. Ann. Med. 2014;46:1-8.

14. Noti M, Sidler D, Brunner T. Extra-adrenal glucocorticoid synthesis in the intestinal epithelium: more than a drop in the ocean? Semin Immunopathol. 2009;31:237-48.

15. Slominski A, Wortsman J. Neuroendocrinology of the skin. Endocr Rev. 2000;21:457-87.

16. Croft AP, O'Callaghan MJ, Shaw SG, Connolly G, Jacquot C, Little HJ. Effects of minor laboratory procedures, adrenalectomy, social defeat or acute alcohol on regional brain concentrations of corticosterone. Brain Res. 2008;1238:12-22.

17. Cima I, Corazza N, Dick B, Fuhrer A, Herren S, Jakob S, et al. Intestinal epithelial cells synthesize glucocorticoids and regulate $\mathrm{T}$ cell activation. J Exp Med. 2004;200:1635-46.

18. Noti M, Corazza N, Tuffin G, Schoonjans K, Brunner T. Lipopolysaccharide induces intestinal glucocorticoid synthesis in a TNFalpha-dependent manner. FASEB J. 2010;24:1340-6.

19. Noti M, Corazza N, Mueller C, Berger B, Brunner T. TNF suppresses acute intestinal inflammation by inducing local glucocorticoid synthesis. J Exp Med. 2010;207:1057-66.

20. Nielsen $\mathrm{OH}$. New strategies for treatment of inflammatory bowel disease. Front Med (Lausanne). 2014;1:3.
21. Stokkers PC, Camoglio L, van Deventer SJ. Tumor necrosis factor (TNF) in inflammatory bowel disease: gene polymorphisms, animal models, and potential for anti-TNF therapy. J Inflamm. 1995;47:97-103.

22. Watts L, Karaderi T, Roberts A, Appleton L, Wordsworth T, Cohen $\mathrm{C}$ et al. The severity of ankylosing spondylitis and responses to anti-tumour necrosis factor biologics are not influenced by the tumour necrosis factor receptor polymorphism incriminated in multiple sclerosis. Genes Immun. 2018. https:// doi.org/10.1038/s41435-018-0017-0. [Epub ahead of print].

23. Botrugno OA, Fayard E, Annicotte JS, Haby C, Brennan T, Wendling O, et al. Synergy between LRH-1 and beta-catenin induces G1 cyclin-mediated cell proliferation. Mol Cell. 2004; 15:499-509.

24. Mueller M, Cima I, Noti M, Fuhrer A, Jakob S, Dubuquoy L, et al. The nuclear receptor LRH-1 critically regulates extra-adrenal glucocorticoid synthesis in the intestine. J Exp Med. 2006;203:2057-62.

25. Coste A, Dubuquoy L, Barnouin R, Annicotte JS, Magnier B, Notti M, et al. LRH-1-mediated glucocorticoid synthesis in enterocytes protects against inflammatory bowel disease. Proc Natl Acad Sci USA. 2007;104:13098-103.

26. Bouguen G, Langlois A, Djouina M, Branche J, Koriche D, Dewaeles E, et al. Intestinal steroidogenesis controls PPARgamma expression in the colon and is impaired during ulcerative colitis. Gut. 2015;64:901-10.

27. Lee JM, Lee YK, Mamrosh JL, Busby SA, Griffin PR, Pathak $\mathrm{MC}$, et al. A nuclear-receptor-dependent phosphatidylcholine pathway with antidiabetic effects. Nature. 2011;474:506U135.

28. Huang J, Jia R, Brunner T. Local synthesis of immunosuppressive glucocorticoids in the intestinal epithelium regulates anti-viral immune responses. Cell Immunol. 2018;334:1-10.

29. Tomlinson JW, Walker EA, Bujalska IJ, Draper N, Lavery GG, Cooper MS, et al. 11beta-hydroxysteroid dehydrogenase type 1: a tissue-specific regulator of glucocorticoid response. Endocr Rev. 2004;25:831-66.

30. Odermatt A, Kratschmar DV. Tissue-specific modulation of mineralocorticoid receptor function by 11 beta-hydroxysteroid dehydrogenases: an overview. Mol Cell Endocrinol. 2012;350:168-86.

31. Schoonjans K, Dubuquoy L, Mebis J, Fayard E, Wendling O, Haby $\mathrm{C}$, et al. Liver receptor homolog 1 contributes to intestinal tumor formation through effects on cell cycle and inflammation. Proc Natl Acad Sci USA. 2005;102:2058-62.

32. Schwaderer J, Gaiser AK, Phan TS, Delgado ME, Brunner T. Liver receptor homolog-1 (NR5a2) regulates CD95/Fas ligand transcription and associated T-cell effector functions. Cell Death Dis. 2017;8:e2745.

33. Lefevre L, Authier H, Stein S, Majorel C, Couderc B, Dardenne $\mathrm{C}$, et al. LRH-1 mediates anti-inflammatory and antifungal phenotype of IL-13-activated macrophages through the PPARgamma ligand synthesis. Nat Commun. 2015;6:6801.

34. Takahashi KI, Fukushima K, Sasano H, Sasaki I, Matsuno S, Krozowski ZS, et al. Type II 11beta-hydroxysteroid dehydrogenase expression in human colonic epithelial cells of inflammatory bowel disease. Dig Dis Sci. 1999;44:2516-22.

35. Stegk JP, Ebert B, Martin HJ, Maser E. Expression profiles of human 11beta-hydroxysteroid dehydrogenases type 1 and type 2 in inflammatory bowel diseases. Mol Cell Endocrinol. 2009;301:104-8.

36. Atanasov AG, Leiser D, Roesselet C, Noti M, Corazza N, Schoonjans $\mathrm{K}$, et al. Cell cycle-dependent regulation of extraadrenal glucocorticoid synthesis in murine intestinal epithelial cells. FASEB J. 2008;22:4117-25. 
37. Sidler D, Renzulli P, Schnoz C, Berger B, Schneider-Jakob S, Fluck $\mathrm{C}$, et al. Colon cancer cells produce immunoregulatory glucocorticoids. Oncogene. 2011;30:2411-9.

38. Mueller M, Atanasov A, Cima I, Corazza N, Schoonjans K, Brunner T. Differential regulation of glucocorticoid synthesis in murine intestinal epithelial versus adrenocortical cell lines. Endocrinology. 2007;148:1445-53.

39. de Souza PR, Sales-Campos H, Basso PJ, Nardini V, Silva A, Banquieri F, et al. Adrenal-derived hormones differentially modulate intestinal immunity in experimental colitis. Mediat Inflamm. 2016;2016:4936370.
40. Huang SC, Lee CT, Chung BC. Tumor necrosis factor suppresses NR5A2 activity and intestinal glucocorticoid synthesis to sustain chronic colitis. Sci Signal. 2014;7:ra20.

41. Krylova IN, Sablin EP, Moore J, Xu RX, Waitt GM, Mackay JA, et al. Structural analyses reveal phosphatidyl inositols as ligands for the NR5 orphan receptors SF-1 and LRH-1. Cell. 2005; 120:343-55.

42. Horino J, Fujimoto M, Terabe F, Serada S, Takahashi T, Soma Y, et al. Suppressor of cytokine signaling-1 ameliorates dextran sulfate sodium-induced colitis in mice. Int Immunol. 2008;20:753-62. 